\title{
Trastocando el derecho a la salud e impulsando la eugenesia
}

\author{
(Comentarios a la resolución
}

del Amparo en Revisión 1388/2015)

\section{Transposing the right to health and boosting eugenesia (Comments to the resolution of the Legal Protection Revisión 1388/2015)}

\author{
Agustín Herrera Fragoso*
}

\section{Resumen}

Hay casos en los que se transforman y trastocan los objetivos de las instituciones por resoluciones y jurisprudencia de los tribunales, situación que se presentó en el análisis de una sentencia del 15 de mayo de 2019, por la $1^{\text {a }}$ Sala de la Suprema Corte de Justicia de la Nación (México), derivado del amparo en revisión 1388/ 2015, por la negativa del hospital de realizar la interrupción del embarazo, por motivos de salud, a pesar de que el niño tendría una vida relativamente normal, perturbando el derecho a la salud, la conciencia social y el valor ético sobre la terapia y de la dignidad humana de todas las personas, discriminando al feto bajo un paradigma eugenésico.

Palabras clave: aborto, derecho a la salud, sentencia, derechos humanos, eugenesia.

\footnotetext{
* Investigador del Instituto de Ciencias Jurídicas de Puebla A.C. y Profesor de la Cátedra de Bioética y Derechos Humanos de la UNESCO, Roma.

Correo electrónico: agusfrag80@hotmail.com

Recibido el 04 de septiembre de 2019. Entregado el 30 de septiembre de 2019.
} 


\section{Introducción}

El 15 de mayo de 2019, la $1^{\text {a }}$ Sala de la Suprema Corte de Justicia de la Nación (SCJN) de México, resolvió el amparo en revisión 1388/2015, por la negativa de un hospital de realizar la interrupción del embarazo de la quejosa, por motivos de salud, por parte de un nosocomio que se rige conforme a la Ley General de Salud y el Código Penal Federal, en donde éste último la quejosa pretende se decrete una discriminación implícita en los artículos 333 y 334 del Código Penal Federal, que tácitamente prohíben la interrupción legal del embarazo por motivos de salud.

Artículo 333.- No es punible el aborto causado sólo por imprudencia de la mujer embarazada, o cuando el embarazo sea resultado de una violación.

Artículo 334.- No se aplicará sanción cuando de no provocarse el aborto, la mujer embarazada o el producto corran peligro de muerte, a juicio del médico que la asista, oyendo éste el dictamen de otro médico, siempre que esto fuera posible y no sea peligrosa la demora.

Se enfatiza que dicha normativa es un acto de concretización de un histórico proceso de discriminación contra las mujeres. Situación que resultó en una sentencia, donde justifica la interrupción del embarazo en concordancia con una eugenesia de un feto con discapacidad, y lo actualiza como un derecho a la salud desde un ámbito liberal.

Por respeto a la quejosa, no se mencionará ninguna alusión y sólo se establecerá un análisis exclusivamente académico del caso en mención.

\section{Antecedentes}

1. En 2013, a la quejosa (en adelante Q), se le informó por parte del personal del nosocomio que se encontraba embarazada: «(...) 
los doctores que la atendieron le hicieron saber que su embarazo era considerado de alto riesgo pues, meses antes, se había sometido a una cirugía de bypass gástrico, tenía 41 años de edad y presentaba un problema de sobrepeso. Por estas razones, Q permaneció internada en el hospital cuatro días, ya que, incluso presentó una amenaza de aborto.

2. Consecutivamente, cuando tenía 15.5 semanas de gestación, Q se sometió a una prueba de amniocentesis genética ${ }^{1}$ con el objetivo de saber si el feto presentaba algún problema hereditario, con los riesgos propios de la técnica. ${ }^{2}$

3. Posteriormente, presentó molestias, sangrado y contracciones. Por esta razón, fue internada de nueva cuenta, al presentar otra amenaza de aborto. Al día siguiente fue dada de alta.

4. Tiempo después, Q recibió los resultados de la amniocentesis genética, que advertían que el feto de sexo masculino presentaba síndrome de Klinefelter. Éste causaría que el feto no pudiera desarrollar sus genitales en la pubertad, pero no impediría que fuera una persona autosuficiente.

5. Dadas todas estas complicaciones «que ocasionan un riesgo a su salud física y emocional», Q solicitó verbalmente y en reiteradas ocasiones a los médicos del hospital que interrumpieran el embarazo.

6. Dadas las reiteradas negativas del personal del hospital, solicitó por escrito la interrupción de su embarazo, en ejercicio de su derecho a la salud y atendiendo a las características de alto riesgo de su embarazo, el cual ponía en riesgo su salud y vida -por su edad y sobrepeso-. Al respecto -entre otros documentos- (...) anexó la opinión técnica del doctor, médico cirujano con especialidad en ginecología y obstetricia. En dicha opinión, el doctor detalló que cursaba un embarazo de alto riesgo, por obesidad grado III, lo que le ocasionaba un riesgo materno mayor de diabetes, tromboembolismo y preeclampsia. A su vez, debido a la cirugía de bypass gástrico, precisó que enfrentaba el riesgo de sufrir malnutrición y la obstrucción del intestino delgado por hernia interna. El 
emisor de la opinión médica recomendó la interrupción del embarazo.

7. Ulteriormente, le realizaron el aborto en una clínica privada a las 19.4 semanas de gestación.

8. Finalmente, por correo, la respuesta de las autoridades responsables fue la negativa a su petición debido a que el feto podría ser autosuficiente, aunque tuviera síndrome de Klinefelter. Además, se indicó que el hospital es una institución de salud del ámbito federal, la cual se rige por la Ley General de Salud, misma que no contempla la interrupción legal del embarazo.

\section{Consideraciones de la quejosa:}

a) La negación de parte de las autoridades responsables de la interrupción del embarazo que mantuvo en riesgo la salud de $\mathrm{Q}, \mathrm{y}$

b) Discriminación por su género, al no ser atendida por su riesgo a la salud de no realizar la interrupción del embarazo.

Posicionamiento en la sentencia por parte de la $1^{a}$ Sala de la SCJN:

1. El caso se debe ver a la luz del parámetro de regularidad constitucional del derecho a la salud y su protección, para luego decidir su aplicación para el caso de una interrupción de embarazo motivada por riesgos a la salud;

2. Es obligación del Estado brindar los servicios de salud preventivos, curativos y paliativos, y abstenerse de imponer prácticas discriminatorias en relación con el estado de salud y las necesidades de las mujeres;

3. La negativa trae una afectación directa del derecho a la salud de $\mathrm{Q}$, al impedirle alcanzar un estado de salud física, psicológica y social integral.

4. La autoridad responsable incumplió su obligación de garantizar el derecho a la salud, pues ignoró las condiciones en que se desarrollaba el embarazo, consistentes en las múltiples amenazas de aborto, la edad de la quejosa, su condición de sobrepeso y de obe- 
sidad mórbida, la condición de primigesta, así como el hecho de saber que el feto tenía el síndrome de Klinefelter, el cual implica sometimiento de por vida a tratamientos médicos y suministro de hormonas y fármacos, lo que definitivamente merma la calidad de vida de cualquier ser humano. La interrupción del embarazo era una alternativa para enfrentar esos riesgos.

5. Las autoridades de salud del hospital debieron ajustar su actuación a una interpretación amplia del derecho a la salud. Para ello, se debió tomar en cuenta lo establecido tanto en la Constitución, como en los tratados internacionales. Las autoridades responsables pertenecientes a un régimen federal deben aplicar un criterio interpretativo del derecho a la salud que brinde una mayor protección a los derechos humanos de las mujeres. Este derecho no puede ser restringido en virtud del lugar de residencia o en atención al régimen local o federal de la institución.

\section{Análisis general}

De los hechos expuestos, se desprende que Q por su edad, obesidad e intervención quirúrgica reciente, al embarazase se expondría a un embarazo de alto riesgo, situación que acorde con la lex artis ad $h o c^{3}$ de la medicina y de la ética médica, debieron informarle y señalarle todas las consecuencias y situaciones que podría padecer por su particular situación de vulnerabilidad, por lo que, si no se realizó, se actualiza una negligencia médica, situación que brinde a toda persona una adecuada información sobre su situación de salud, respetando su autonomía, libertad y en particular su dignidad humana.

Por otro lado, a pesar de que $Q$ ya había tenido una primera dificultad de aborto espontáneo, solicitó posteriormente una prueba de amniocentesis genética con el objetivo de saber si el feto presentaba algún problema congénito, situación que aumentaba el riesgo a su salud y la posibilidad de sufrir un aborto, por lo que 
posteriormente presentó molestias y sangrado; de lo cual debió haberse llevado un consentimiento informado de acuerdo con su situación; asimismo, se debió brindarle toda la asesoría y acompañamiento psicosocial, para la atención que requería.

Posteriormente, con el resultado de la amniocentesis y diagnóstico de síndrome de Klinefelter, solicitó que se le practicara la interrupción del embarazo, situación que implica un acto eugenésico.

Puede deducirse que probablemente hubo una falta de ética y profesional, si los profesionales de la salud no le expusieron una adecuada y correcta información, sobre la consecuencias y alternativas y apoyo que se le puede brindar para un adecuado seguimiento y atención de su hijo.

Sin embargo, la institución de seguridad social no accedió a la petición de aborto, siguiendo la estricta legalidad de las normas que le rigen, así como no existir las condiciones de realizarlo, ya que lo que existía era un embarazo de alto riesgo.

Pero como la propia amniocentesis podría haberle provocado un aborto, puede considerarse una negligencia médica.

\section{Análisis de fondo}

1. El pronunciamiento jurídico de la Sentencia basa su estructura sobre el parámetro de control de regularidad constitucional, reconociendo única y exclusivamente la solicitud de la quejosa;

2. Se establece que se violó el derecho a la salud en su enfoque de derecho a la salud reproductiva y que se discriminó por su situación de mujer, debiéndole brindar el aborto por su violación a su salud sexual y reproductiva, y

3. Se abundó sobre el derecho a su bienestar y proyecto de vida, señalando la importancia de las expectativas que cada persona tiene para su vida de acuerdo con sus condiciones y su contexto, teniendo como fundamento la autodeterminación de cómo cada mujer quiere vivir su vida. 


\section{Párrafos preocupantes de la sentencia}

a) «No basta con tener libertad para adoptar, autónomamente, las decisiones acerca de la propia salud; es fundamental poder ejecutarlas adecuadamente. Esto es, la decisión sobre la propia salud, como terminar un embarazo.»

b) «Asiste razón a la quejosa cuando afirma que las autoridades responsables no evaluaron su salud en términos integrales y que se concentraron en destacar que el síndrome de Klinefelter detectado al feto no era incompatible con una vida independiente, ignorando la importancia de la salud física, emocional y social de la señora.»

c) «Se comparte el argumento de la quejosa en cuanto a que la negativa y la dilación subsecuente de las autoridades señaladas como responsables constituyeron formas de trato cruel, inhumano y degradante.»

d) «Las mujeres están ubicadas en una situación de dependencia y vulnerabilidad, que condiciona que sus objetivos de salud sólo puedan conseguirse si este sistema les facilita dichos servicios. Por tanto, los prestadores de servicios de salud tienen la decisión final sobre la integridad personal de las mujeres; en especial, en el caso del aborto terapéutico donde forzarla a continuar un embarazo, genera per se un daño sobre la salud de la mujer, independientemente del momento en que éste se interrumpa.»

e) «Fue incorrecto que la autoridad responsable argumentara que el síndrome detectado al feto no era incompatible con una vida sana, pues tal manifestación no es congruente con la solicitud que se le planteó. En ese sentido, la autoridad debió pronunciarse sobre el derecho a la salud de la mujer, el cual es un derecho tutelado expresamente por la Constitución y los tratados internacionales de derechos humanos. Sobre todo, porque la señora Q tenía un embarazo de alto riesgo por tener 40 años de edad, haberse practicado previamente un «bypass gástrico» y por haber padecido una amenaza de aborto previamente. Así, las autoridades debieron considerar estas afectaciones a la salud, pues es constitucionalmente 
inaceptable que la salud de la mujer se ponga en riesgo por la continuidad de un embarazo a una edad avanzada y con un historial clínico de esa naturaleza. La decisión de interrumpir un embarazo le corresponde única y exclusivamente a la mujer que lo cursa...»

f) «Fue víctima en reiteradas ocasiones de maltrato psicológico por parte de las autoridades que la atendieron en el centro de salud, ya que en las diversas ocasiones en las que les expresó su preocupación y angustia por las implicaciones que la continuación de un embarazo de un feto con el síndrome diagnosticado tendría en su salud y calidad de vida, los médicos respondieron con argumentos que descalificaron lo referido por ella e, incluso, cuestionaron el ejercicio de su libertad sexual. Así como la negativa a realizar la interrupción del embarazo.»

\section{Comentarios}

i) Es preocupante el relativismo que se expone sobre la vida humana, basado en la condición del feto respecto a una discapacidad; esto es que Q, al enterarse del padecimiento de su hijo (síndrome de Klinefelter), solicita terminar con su embarazo, mismo que, desafortunadamente, está justificado en la sentencia.

ii) El síndrome de Klinefelter. ${ }^{4}$ Nombres alternativos: Síndrome 47 X-X-Y; Síndrome XXY; Trisomía XXY; 47,XXY/46,XY; Síndrome de mosaico; Síndrome Poli x Klinefelter. ${ }^{5}$

Es una afección genética que sucede en los hombres cuando tienen un cromosoma $\mathrm{X}$ extra, con lo cual se presenta en aproximadamente 1 de cada 500 a 1,000 bebés varones. Las mujeres que resultan embarazadas después de los 35 años tienen una probabilidad ligeramente mayor de tener un niño con este síndrome que las mujeres más jóvenes.

Los síntomas que presentan son:

1. La infertilidad;

2. Proporciones corporales anormales (piernas largas, tronco corto, hombro igual al tamaño de la cadera); 
3. Agrandamiento anormal de las mamas (ginecomastia);

4. Problemas sexuales;

5. Vello púbico, axilar y facial menor a la cantidad normal;

6. Testículos pequeños y firmes;

7. Estatura alta, $y$

8. Tamaño reducido del pene.

A estas personas se les puede recetar terapia con testosterona y puede ayudar a:

a) Promover el crecimiento de vello corporal;

b) Mejorar la apariencia de los músculos;

c) Mejorar la concentración;

d) Mejorar la autoestima y el estado de ánimo;

e) Mejorar la energía y el impulso sexual, y

f) Mejorar la fuerza.

La mayoría de los hombres con este síndrome no son capaces de embarazar a una mujer. Pero, un especialista en infertilidad puede ayudarlos. Visitar a un endocrinólogo también puede ser útil. A estas personas siempre se les debe canalizar con grupos de apoyo. ${ }^{6}$

Posibles complicaciones: el agrandamiento de los dientes con un adelgazamiento de la superficie es muy común en el síndrome de Klinefelter. Esto se conoce como taurodontismo. Se puede observar en radiografías dentales.

Este síndrome también incrementa el riesgo de:

i. Trastorno de hiperactividad y déficit de atención (THDA).

ii. Trastornos autoinmunitarios como el lupus, la artritis reumatoidea y el síndrome de Sjögren.

iii. Cáncer de mama en hombres.

iv. Depresión.

v. Dificultades de aprendizaje, incluso dislexia, que afecta la lectura. 
vi. Un raro tipo de tumor llamado células germinativas extragonadales.

vii. Enfermedad pulmonar.

viii. Osteoporosis.

ix. Venas varicosas.

Las personas con tal discapacidad pueden vivir con apoyo y un buen trato humano; no es dable exterminarlos, validando un acto de discriminación y eugenesia positiva. A mayor abundamiento, el falaz argumento de provocar la eugenesia para tener una vida digna o para evitar el sufrimiento o dolor de las mujeres para llegar a un embarazo a término, o de no permitir que nazcan personas con discapacidad, por no tener una opción de vida digna, además de ser un mecanismo de defensa psicológica de quien lo aduce, tienen una premisa falsa. Las personas con discapacidad, per se, no nacen infelices, ni se hacen infelices por su discapacidad, especialmente si es genética. Es la predisposición psicológica adulta a que la sentencia de infelicidad se cumpla. Si se parte aun inconscientemente de ser infeliz, se acaban realizando actos que harán que, efectivamente, esa persona sea infeliz. Este hecho, está bien constatado en toda la psicología del desarrollo infantil. ${ }^{7}$

En cambio, la actitud de aceptación incondicional, esto es, amorosa, parte donde se hace todo lo que pueda para ser feliz y con ello apoya significativamente al desarrollo de una personalidad fuerte y madura de estas personas. Por ejemplo, el que se elimine o discrimine por tener una discapacidad como síndrome de Down (Trisomía 21), con síndrome de Turner (Monosomía X), o Klinefelter (Trisomía XXY), donde la mayoría de los personas llevan una vida normal y productiva; más aún, los grandes humanistas Nicholas James Vujic y Hirotada Ototake, con el síndrome de Tetra-Amelia, nacieron sin piernas ni brazos, y según la ideología de perfección, serían candidatos idóneos para el aborto; sin embargo y a contrario sensu, siguiendo la lex artis ad hoc, se deben de brindar los tratamientos y apoyos necesarios para su inclusión. 
El querer fundamentar la muerte de estas personas en la primera etapa de su existencia es otorgar un falso derecho, son mero ejercicio de la ley del más fuerte, que bien puede ser una mayoría de consensos, de expertos, parlamentaria democráticamente elegida o una decisión jurisdiccional de uno o unos cuantos -como es el caso-. Es puro arbitrio de los que ostentan el poder, que sancionan su supuesto derecho a pisotear al más débil, que en este caso carece de toda posibilidad de defensa. Representantes que no pueden ser la fuente última del bien y del mal.

El actual modelo social considera que el problema no está en la persona con discapacidad, sino en los factores sociales que generan su exclusión. En la medida en que desaparecen las barreras que impiden a las personas con discapacidad llevar una vida autónoma y tener las mismas oportunidades que el resto de ciudadanos, la discapacidad deja de ser un factor de exclusión. Así entendida la discapacidad, deja de percibirse como una desgracia y se contempla como una manifestación más de la fragilidad humana que no debe impedir, en tanto sea posible, tener una vida plena. Aceptar el aborto por el riesgo de una futura discapacidad, supone la regresión a una concepción de la discapacidad anacrónica y, sobre todo, discriminatoria.

Sobre este criterio, en 2011 cinco agencias de Naciones Unidas ${ }^{8}$ elaboraron conjuntamente un informe para denunciar la práctica de seleccionar el sexo de las personas que van a nacer mediante abortos selectivos, extendida en muchos países del mundo. En el mismo año, el Comité de Derechos de las Personas con Discapacidad hizo públicas unas consideraciones sobre el informe presentado por España acerca del grado de cumplimiento de la Convención de los Derechos de las Personas con Discapacidad, donde reprocha a España el tratamiento que hace de la discapacidad en la regulación vigente sobre el aborto. Sostiene que cada Estado es libre para establecer su propia regulación sobre el aborto, pero rechaza que una razón para considerar lícito el aborto sea la discapacidad. ' Sobre este particular, destaca que los Países Bajos en 
el momento de firmar la Convención sobre los derechos de las personas con discapacidad y a propósito del artículo 10 «Derecho a la vida», los Estados parte reafirman el derecho inherente a la vida de todos los seres humanos y adoptarán todas las medidas necesarias para garantizar el goce efectivo de ese derecho por las personas con discapacidad en igualdad de condiciones con los demás. ${ }^{10}$

Sobre el derecho a la salud bajo el parámetro de control de regularidad constitucional, ${ }^{11}$ es de destacar:

Como se puede apreciar en el concepto del «más alto nivel posible de salud», a que se hace referencia en el párrafo $1^{\circ}$ del artículo 12 del Pacto Internacional de Derechos Económicos Sociales y Culturales (PIDESC), se toman en cuenta tanto las condiciones biológicas y socioeconómicas esenciales de la persona como los recursos con que cuenta el Estado, y que existen varios aspectos que no pueden abordarse únicamente desde el punto de vista de la relación entre el Estado y los individuos; en particular, un Estado no puede garantizar la buena salud ni puede brindar protección contra todas las causas posibles de la mala salud del ser humano. Así, los factores genéticos, la propensión individual a una afección y la adopción de estilos de vida malsanos o arriesgados suelen desempeñar un papel importante en lo que respecta a la salud de la persona. Por lo tanto, el derecho a la salud debe entenderse como un derecho al disfrute de toda una gama de facilidades, bienes, servicios y condiciones necesarios para alcanzar el más alto nivel posible de salud.

Lo que ha precisado la Organización Mundial de la Salud (OMS), en el sentido de que la salud es un estado de completo bienestar físico, mental y social y no solamente la ausencia de afecciones o de enfermedades, y que el goce del máximo grado de salud que se pueda lograr es uno de los derechos fundamentales de todo ser humano sin distinción de raza, religión, ideología política o condición económica o social. ${ }^{12}$ A partir de esa definición, bien- 
estar $^{13}$ se convierte en el concepto más amplio de salud, los cambios en el estado del medio ambiente pueden redundar en impactos ambientales, capaces de influenciar positiva o negativamente servicios ambientales que determinan el bienestar humano. Por ejemplo, los efectos a la salud asociados a la degradación o pérdida de servicios ecosistémicos incluyen: mortalidad evitable; ${ }^{14}$ morbilidad $;{ }^{15}$ carga de enfermedad $;{ }^{16}$ malestar/sufrimiento psicológico ${ }^{17}$ y violencia (física o simbólica). ${ }^{18}$

Entonces, la salud involucra actividades de prevención, promoción y protección e implica un enfoque integral en donde se incluyen los entornos físico y social, así como los demás factores relacionados con la existencia. Bajo un enfoque similar y de forma muy interesante dicho derecho lo define la parte última de la última tesis de la Suprema Corte citada en el proyecto: «[...] el derecho a la salud debe entenderse como un derecho al disfrute de toda una gama de facilidades, bienes, servicios y condiciones necesarios para alcanzar el más alto nivel posible de salud.» El mismo Organo también señala: «el derecho a la salud, que implica la obtención de un determinado bienestar general y que se integra necesariamente por el estado físico, mental, emocional y social del sujeto, incluyendo entonces el derecho a la integridad físico-psicológica». ${ }^{19}$

Como se advierte, el tema de bienestar y salud es totalmente diferente a como lo exponen tanto la quejosa como la sentencia. Por su parte, el Comité de Derechos Económicos, Sociales y Culturales (Observación General 14, párr. 36) establece que existe la obligación de garantizar que la infraestructura de la sanidad pública proporcione servicios de salud sexual y genésica, incluida la maternidad segura, sobre todo en las zonas rurales.

En esa inteligencia, la Observación General 15 de los derechos del niño al disfrute del más alto nivel posible de salud (art. 24), el párrafo 18 señala: «Entre los principales determinantes de la salud, la nutrición y el desarrollo del niño, cabe mencionar la realización 
del derecho de la madre a la salud y el papel de los progenitores y otros cuidadores [...]».

Por lo que el derecho a la salud, en ningún momento fomenta el aborto o la mal llamada interrupción del embarazo.

Con base en el derecho a la salud y a la mujer, un embarazo de alto riesgo es el que tiene factores asociados que pueden afectar negativamente a la salud de la madre o del feto. Un buen control de la gestación resulta clave para evitar problemas mayores, como debe ser con un enfoque de salud.

A lo que un embarazo de alto riesgo es aquel que tiene más posibilidades de complicaciones, tanto desde el punto de vista de la madre como para el bebé y, por tanto, el control previo durante la gestación, el parto y puerperio han de ser más completos, evitando cualquier posible riesgo.

El término se refiere a que circunstancias médicas, sociales, ginecológicas u obstétricas puedan poner en riesgo la salud de la madre, del bebé, o de ambos, con una probabilidad superior a la de la población general durante la gestación, el parto o el puerperio.

Las causas del embarazo de alto riesgo son muy variadas, pero existen una serie de factores asociados al mismo, algunos de los cuales están presentes antes de que la mujer quede embarazada, mientras que otros se desarrollan durante la gestación. Es importante identificarlos precozmente (idealmente antes de que se produzca la concepción) para estimar su importancia y disminuir así las consecuencias adversas de los mismos, ya que aumentan tanto la incidencia de complicaciones durante el embarazo, como el riesgo de que la situación se repita en gestaciones posteriores, para el caso que nos ocupa:

- Antecedentes sociales: mujeres mayores de 40 años; con sobrepeso (IMC mayor de 35);

- Antecedentes médicos: enfermedades crónicas como tensión arterial alta, problemas del corazón, alteraciones del metabolismo (obesidad), bypas gástrico, y

- Patología en el embarazo actual. 
Trastocando el derecho a la salud e impulsando la eugenesia

El hecho de identificar un embarazo como de alto riesgo, antes o durante su transcurso, asegura que la mujer ha de llevar un seguimiento más completo y una serie de cuidados especiales, no un aborto como solución.

En el presente caso, y en los subsecuentes, desde una perspectiva bioética y biojurídica y, bajo los estándares de derechos humanos, respetando a todas las personas por su dignidad ontológica, y sin discriminación por sexo, salud, edad o cualquier otra distinción que lo excluya como otro más de nuestra especie y común humanidad; debiendo prever, como medida preventiva y en el contexto del derecho a la salud, es la consulta preconcepción, recomendable antes de planificar un embarazo, con la finalidad de identificar las condiciones sociales y médicas, tanto maternas como paternas, que puedan ser optimizadas antes de la gestación, con el fin de incrementar las posibilidades de un resultado perinatal favorable.

En la primera consulta se realizará una historia clínica completa, solicitud de pruebas básicas y otras que nos ayuden al control de la patología propia de cada paciente, y una exploración ginecológica y mamaria con toma de citología cervical según el programa de cribado poblacional.

Asimismo, se debe incidir en un protocolo más individualizado de seguimiento, con un mayor número de visitas con el tocólogo, para controlar la evolución y realizar un diagnóstico precoz de las complicaciones. La frecuencia de cada visita y exploraciones vendrán determinadas por el tipo y severidad de la enfermedad, intercalando con las visitas a la matrona.

Siempre habrá que controlar: peso, tensión arterial, edemas o varices, maniobras de Leopold (presentación y encajamiento del bebé), medición de altura uterina, auscultación cardiaca fetal (a partir del segundo trimestre), detección de dinámica uterina (contracciones) (a partir del tercer trimestre), exploración cervical (en segundo-tercer trimestre y en función de antecedentes), y ecografías (estudio ecográfico y doppler fetal por una Unidad de Diagnóstico Prenatal). 
En algunos casos es necesario permanecer un tiempo ingresada en el hospital para tratar posibles complicaciones que surjan tras dar a luz. A veces es preciso que otros especialistas médicos valoren el estado de la madre. Se recomienda también que la embarazada debe ser tratada antes, durante y después del parto, en un Hospital de III Nivel o especializado en Medicina Materno-Fetal con Unidad de Cuidados Intensivos Neonatal y de adultos, en caso de complicaciones.

Un hecho importante en el control del embarazo de alto riesgo obstétrico es mantener a la paciente siempre informada del pronóstico de su embarazo, la influencia de la gestación en su enfermedad, las posibles complicaciones y su prevención, la frecuencia de visitas, los signos de alarma ante los que deberá acudir al hospital, y la vía y el momento de finalización del embarazo, situación que por ningún motivo es un trato cruel inhumano o degradante; más bien es un consentimiento informado y un seguimiento de atención y acompañamiento de acuerdo con su situación particular.

Si el embarazo está bien controlado y se siguen las recomendaciones del médico no deben de surgir complicaciones. Cuando una mujer tiene un embarazo de alto riesgo es conveniente que adopte una serie de medidas para prevenir las complicaciones en lo posible, no fomentar el aborto, de lo cual así sí se cumple un derecho a su salud sexual y reproductiva, con perspectiva humana y acorde con la lex artis ad hoc de la medicina.

\section{Conclusión}

Por lo señalado, lo que se vislumbra es la exclusión del feto, negando su dignidad humana, valor fundamental e inherente de todo ser humano, desconociendo su valor como persona y, en consecuencia, cualquier derecho reconocido por la constitución y tratado en materia de derechos humanos. 
Al realizar la prueba de amniocentesis genética que determina el síndrome de Klinefelter, se actualiza una eugenesia liberal, violando el principio de sindéresis, núcleo de la ética y derecho «haz el bien y evita el mal», y violando la coexistencia relacional de todas las personas, por una resolución injusta y discriminatoria, en contra del feto por su discapacidad, negando su humanidad por excluirlo dentro del género humano y, más aún, lo estigmatiza como indeseable y dándole a su madre el derecho a eliminarlo de su existencia, rebajándolo a objeto de derecho, situación que la SCJN avala y justifica, violando los principios de exhaustividad e imparcialidad, impulsando y generando un antecedente jurisdiccional tendente a la eugenesia, situación que va en contra de la ética, bioética y biojurídica, al establecer criterios que cosifican y empiezan a establecer referentes que discriminan a los seres humanos al inicio de su existencia, incrementando la vulnerabilidad e invisibilizando a los embriones y fetos.

Lo cual es un peligro, para el relativismo que nos invade y más aún en los albores del trans y poshumanismo, que amenazan con discriminar a los seres humanos que no cumplan los estándares de calidad y bienestar de vida.

Aquí se corrompe el bien y correcta atención y seguimiento terapéutico que deben tener todas las personas en cualquier momento de su existencia, bajo los principios éticos de totalidad, terapéutico y de subsidiariedad que deben seguir todos los profesionales de la salud, mismos que deben reconocer los órganos jurisdiccionales en beneficio de la vida, integridad personal y salud de todas las personas en cualquier momento de su existencia sin distinción alguna.

Ahora bien, si los profesionales de la salud siguieron todos los protocolos para una adecuada e informada atención materno infantil, y el pronunciamiento es constructivo y cooperativo para todas las personas, sin discriminación de ningún tipo y no sólo establecer como única opción el del aborto, bajo falacias y eufemismos fuera de la buena práctica médica y, más aún, bajo criterios basados 
en evidencia y con principios éticos y bioéticos, el camino es claro para la salvaguarda de la vida y salud de todas las personas relacionadas al caso.

Por último, un tribunal constitucional tiene que analizar el caso sobre todas las personas implicadas y más aún en una situación de vulnerabilidad; asimismo, si el parámetro de regularidad constitucional del derecho a la salud es el referente de aplicación, se debe velar por todos los pacientes y por la correcta atención médica de acuerdo con la lex artis ad hoc, de manera que se realicen todos los procedimientos avalados y estandarizados por instancias profesionales y acordes a la ciencia, basada en evidencia y principios éticos, sin ningún tipo de discriminación y bajo los valores jurídicos que deben respetar la ontología del ser humano, base de la biojurídica.

\section{Referencias bibliográficas}

${ }^{1}$ Puede tener riesgo de aborto.

2 Estudio citogenético, prenatal.

${ }^{3}$ «Aquel criterio valorativo de la corrección del concreto acto médico ejecutado por el profesional de la medicina-ciencia o arte médico que tiene en cuenta las especiales características de su autor, de la profesión, de la complejidad y trascendencia vital del paciente y, en su caso, de la influencia en otros factores endógenos -estado e intervención del enfermo, de sus familiares, o de la misma organización sanitaria-, para calificar dicho acto de conforme o no con la técnica normal requerida (derivando de ello tanto el acervo de exigencias o requisitos de legitimación o actuación lícita, de la correspondiente eficacia de los servicios prestados y, en particular, de la posible responsabilidad de su autor/médico por el resultado de su intervención o acto médico ejecutado)». T.A. (10ª) Tribunales Colegiados, I.4o.A.92 A, Libro XXV, octubre de 2013, Tomo 3.

${ }^{4}$ AlLAN CA, McLaCHLAN RI. Androgen deficiency disorders. In: Jameson JL, De Groot LJ, de Kretser DM, et al, eds. Endocrinology: Adult and Pediatric. 7th ed. Philadelphia, PA: Elsevier Saunders; 2016: chap 139; Matsumoto AM, Bremmer WJ. Testicular disorders. In: Melmed S, Polonsky KS, Larsen PR, Kronenberg HM, eds. Williams Textbook of Endocrinology. 13th ed. Philadelphia, PA: Elsevier; 2016: chap 19; Nussbaum RL, Mclnnes RR, Willard HF. The chromosomal and genomic basis of disease. In: Nussbaum RL, Mclnnes RR, Willard HF, eds. Thompson \& Thompson Genetics in Medicine. 8th ed. Philadelphia, PA: Elsevier; 2016: chap 6. 
${ }^{5}$ Es un trastorno autoinmunitario en el cual se destruyen las glándulas que producen las lágrimas y la saliva, lo que causa resequedad en la boca y en los ojos. Este trastorno puede afectar a otras partes del cuerpo, incluso los riñones y los pulmones.

${ }^{6}$ En E.U.: American Association for Klinefelter Syndrome Information and Support (AAKSIS) - www.aaksis.org.

7 CRAIG, Grace. Desarrollo psicológico. Prentice may. México. Séptima edición 2000, p. 175.

${ }^{8} \mathrm{OHCHR}$, UNFPA, UNICEF, UN Women and WHO, Preventing gender-biased sex selection. An interagency statement, Ginebra, 2011.

9 «17. The Committee takes note of Act 2/2010 of 3 March 2010 on sexual and reproductive health, which decriminalizes voluntary termination of pregnancy, allows pregnancy to be terminated up to 14 weeks and includes two specific cases in which the time limits for abortion are extended if the foetus has a disability: until 22 weeks of gestation, provided there is "a risk of serious anomalies in the foetus", and beyond week 22 when, inter alia, "an extremely serious and incurable illness is detected in the foetus". The Committee also notes the explanations provided by the State party for maintaining this distinction. 18. The Committee recommends that the State party abolish the distinction made in Act 2/2010 in the period allowed under law within which a pregnancy can be terminated based solely on disability».

${ }^{10}$ Convención de la ONU sobre los derechos de todas las personas con discapacidad, 2006, art. 10.

${ }^{11}$ El primer párrafo del artículo $1^{\circ}$ constitucional reconoce un conjunto de derechos humanos cuyas fuentes son la Constitución y los tratados internacionales de los cuales el Estado Mexicano sea parte.

12 Principios básicos de la Constitución de la Organización Mundial de la Salud. La Constitución fue adoptada por la Conferencia Sanitaria Internacional, celebrada en Nueva York del 19 de junio al 22 de julio de 1946, y firmada el 22 de julio de 1946 por los representantes de 61 Estados.

${ }^{13}$ La Evaluación de Ecosistemas del Milenio ha actualizado el concepto «bienestar», relacionándolo con los servicios de los ecosistemas. En esta perspectiva, el bienestar humano está compuesto por cinco componentes principales interrelacionados: las bases materiales para una buena vida, la salud, las buenas relaciones sociales, la seguridad y la libertad de elección y de acción. Los componentes del bienestar humano están particularmente determinados por la integridad de los ecosistemas para brindar servicios ambientales. También dependen de la educación y de las garantías de acceso a servicios humanos de calidad (OMS, 2005).

${ }^{14}$ La mortalidad evitable es un efecto a la salud que debe diferenciarse de la mortalidad como fenómeno demográfico. Las causas de mortalidad evitable asociadas a impactos ambientales afectan principalmente la infancia.

${ }^{15}$ La morbilidad se refiere a la incidencia de patologías definidas y puede evaluarse de diversas formas (número de casos registrados; números de internaciones o de consultas médicas).

Medicina y Ética - Octubre-Diciembre 2019 - Vol. 30 - Núm. 4 


\section{A. Herrera Fragoso}

${ }^{16}$ La carga de enfermedad incluye los años de vida perdidos por muerte prematura y los años de vida vividos con discapacidad.

${ }^{17}$ El malestar y el sufrimiento psicológico son fenómenos subjetivos que pueden estar determinados por impactos ambientales, tales como las pérdidas estéticas 0 materiales (paisaje, entorno) y de arraigo (migración forzosa).

${ }^{18}$ La violencia (física o simbólica) está asociada a la pérdida de las buenas relaciones sociales (cohesión social, respeto mutuo, justicia social).

${ }^{19}$ Amparo Directo Civil 6/2008 del 6 de enero de 2009. 